\title{
The impact of comorbid depression-diabetes on proteomic outcomes among community-dwelling Mexican Americans with mild cognitive impairment
}

\author{
Leigh Ann Johnson, ${ }^{1}$ Fan Zhang, ${ }^{2}$ Stephanie Large, James Hall, \\ and Sidney E. O'Bryant ${ }^{\top}$ \\ ${ }^{1}$ Department of Pharmacology and Neuroscience, Institute for Translational Research, University of North Texas Health Science Center, Fort Worth, TX, USA \\ ${ }^{2}$ Department of Biology, University of Vermont, Burlington, VT, USA
}

ABSTRACT

Background: Mexican Americans suffer from a disproportionate burden of modifiable risk factors, which may contribute to the health disparities in mild cognitive impairment (MCI) and Alzheimer's disease (AD).

Objective: The purpose of this study was to elucidate the impact of comorbid depression and diabetes on proteomic outcomes among community-dwelling Mexican American adults and elders.

Methods: Data from participants enrolled in the Health and Aging Brain among Latino Elders study was utilized. Participants were 50 or older and identified as Mexican American $(N=514)$. Cognition was assessed via neuropsychological test battery and diagnoses of MCI and $\mathrm{AD}$ adjudicated by consensus review. The sample was stratified into four groups: Depression only, Neither depression nor diabetes, Diabetes only, and Comorbid depression and diabetes. Proteomic profiles were created via support vector machine analyses.

Results: In Mexican Americans, the proteomic profile of MCI may change based upon the presence of diabetes. The profile has a strong inflammatory component and diabetes increases metabolic markers in the profile.

Conclusion: Medical comorbidities may impact the proteomics of MCI and $\mathrm{AD}$, which lend support for a precision medicine approach to treating this disease.

Key words: Mexican Americans, diabetes mellitus, depression, proteomics

\section{Introduction}

Alzheimer's disease (AD) is the most common neurodegenerative dementia, and over 5.7 million Americans are living with a diagnosis of $\mathrm{AD}$ (Alzheimer's Association, 2017). AD is the sixth leading cause of death in the U.S.A., and the costs associated with care for the disease are estimated to be $\$ 277$ billion (Alzheimer's Association, 2017). As the population ages, the prevalence of $\mathrm{AD}$ is expected to grow dramatically, with estimates reaching up to 14 million by 2050 (Alzheimer's Association, 2017). Mexican Americans are one of the fastest aging populations in the U.S.A. and are at increased risk of developing $\mathrm{AD}$ or mild cognitive impairment (MCI) (Alzheimer's

Correspondence should be addressed to: Leigh Ann Johnson, Department of Pharmacology and Neuroscience, University of North Texas Health Science Center, 3500 Camp Bowie Blvd, Fort Worth, TX 76107, USA. Phone: +817735-2965. Email: leigh.johnson@unthsc.edu. Received 14 Jun 2019; revision requested 05 Sep 2019; revised version received 13 Sep 2019; accepted 23 Sep 2019. First published online 29 October 2019.
Association, 2017; Jacobsen et al., 2011; Novak and Riggs, 2004). Mexican Americans develop MCI and $\mathrm{AD}$ at younger ages and when they are diagnosed with cognitive impairment, they are diagnosed at more advanced stages. (O'bryant et al., 2007; 2013a; 2013b). Mexican Americans have higher rates of modifiable risk factors such as diabetes and depression have a lower frequency of the ApoE\&4 allele as well as demonstrating an $\mathrm{AD}$ proteomic profile that is metabolic in nature (Haan et al., 2003; O'bryant et al., 2013a; 2013b; 2013c; Sundquist and Winkleby, 1999). Despite the demonstrated medical, genetic, and proteomic differences among Mexican Americans diagnosed with $\mathrm{MCI}$ and $\mathrm{AD}$ as compared to non-Hispanic whites, there is a dearth of literature investigating biological mechanisms and pathways for $\mathrm{AD}$ among this group (O'Bryant et al., 2010; 2013c; 2013d). Therefore, the goal of this study was to elucidate the impact of comorbid depression and diabetes on proteomic outcomes among community-dwelling Mexican American adults and elders. 
Depression and diabetes mellitus (DM) are two $\mathrm{AD}$ risk factors which are more prevalent among Mexicans Americans. Individually, both depression and diabetes have been linked to cognitive decline. Multiple epidemiological studies such as the Rotterdam Study, the Framingham Heart Study, the Honolulu-Asia Aging Study, and the Religious Orders Study have found DM increased the risk for $\mathrm{AD}, \mathrm{MCI}$, and cognitive dysfunction (Arvanitakis et al., 2004; Elias et al., 2005; Ott et al., 1996; 1999; Peila et al., 2002). Mexican Americans diagnosed with $\mathrm{MCI}$ and $\mathrm{AD}$ consistently have higher rates of type two DM (Gerst et al., 2010; Luchsinger et al., 2007; Palmer et al., 1996) and metabolic factors have been consistently strongly related to MCI among Mexican Americans (O'Bryant et al., 2013a; Palmer et al., 1996). Research indicates type two DM impacts some of the basic pathological mechanisms of AD (de la Monte, 2014; Kandimalla et al., 2017). For example, insulin plays a role in the phosphorylation of tau and the formation of amyloid plaques, and insulin resistance has been hypothesized as a mechanism for cognitive decline (de la Monte, 2014; Kandimalla et al., 2017). Both AD and type two DM are characterized by brain atrophy, reduced cerebral glucose metabolism, and insulin resistance (Verdile et al., 2015).

Depression, which is highly prevalent in Mexican Americans, is a modifiable risk factor for MCI and AD. Prior work has demonstrated that Mexican Americans suffer significantly higher rates of depression chronicity in the USA and significant gaps exist between depression diagnosis and treatment when compared to non-Hispanic whites (González et al., 2010; Hinton et al., 2012). Inflammation has been proposed as a biological pathway for the development of depression (Smith et al., 2018). In a population-based study in Rotterdam, higher IL6 (Interleukin 6) levels were strongly associated with depression among adults aged 60 years. Of 1,686 participants aged 70 years and above from the Duke Established Population for Epidemiologic Studies of the Elderly, serum IL6 was significantly associated with depression. In a recent meta-analysis, both IL-6 and CRP (C-reactive protein) were found to be associated with depression among older adults, and longitudinal data suggested that inflammation leads to depression rather than depression leading to inflammation (Smith et al., 2018; Tiemeier et al., 2013). A possible mechanism of action is that proinflammatory cytokines are able to cross the bloodbrain barrier and can affect structures such as the amygdala that regulate emotions (Smith et al., 2018). Depression increases risk for progression from $\mathrm{MCI}$ to $\mathrm{AD}$, as well as risk for incident MCI over time (Barnes et al., 2006; Modrego and Ferrandez, 2004).
Both depression and diabetes are prevalent and often co-occurring conditions in the elderly, and researchers have found this comorbidity significantly increased risk for MCI and AD across multiple cohorts (Downer et al., 2016). However, the majority of this research has been conducted among non-Hispanic populations. The depression-diabetes comorbidity was associated with consistently increased risk for $\mathrm{MCI}$ and $\mathrm{AD}$ in Mexican Americans, but not non-Hispanic whites (Johnson et al., 2015). When examining proteomic profiles indicative of $\mathrm{AD}$, our work has found that the proteomic profile of AD among Mexican Americans appears to be largely metabolic in nature as compared to a more inflammatory/vascular-weighted $\mathrm{AD}$ profile found among non-Hispanic whites (O'Bryant et al., 2013a; 2013b). Again, when looking at proteomic markers, a combination of elevated depression and inflammation has been found to be associated with poorer memory performance among Mexican Americans. This work suggests that (1) depression and diabetes may be particularly important risk factors for MCI and $\mathrm{AD}$ among Mexican Americans, (2) there are proteomic differences in $\mathrm{AD}$ among Mexican Americans as compared to nonHispanic whites, (3) Depression in combination with inflammation may further increase risk for cognitive loss, and (4) inflammation may play a significant role in MCI among Mexican Americans, whereas a metabolic shift may occur in the transition to $\mathrm{AD}$ where the profile becomes more metabolic nature. In this study, we sought to examine the impact of depression and diabetes (alone and in combination) on the proteomic profile of MCI among Mexican Americans. An understanding of depression and diabetes can have significant implications as to which biological pathways are impacted by these conditions. These comorbid conditions may affect the interpretation of proteomic profiles associated with cognitive loss and MCI among Mexican Americans.

\section{Materials and methods}

\section{Participants}

Data from 515 participants from the Health and Aging Brain among Latino Elders (HABLE) study were analyzed. The HABLE study is a communitybased, epidemiological study of cognitive aging among Mexican American adults and elders. Additional recruitment methods include placing ads in local newspapers, distributing flyers and brochures through our community partners, snowball recruitment, and attendance at health fairs. Each participant underwent an interview (demographics, medical history, health behaviors), neuropsychological testing, 
fasting blood draw, and a medical examination. Additionally, all participants were required to name an informant that was willing and able to answer questions regarding their activities of daily living and cognition. Participants were interviewed in either English or Spanish, based on their preference. Cognitive diagnoses of MCI were assigned according to Mayo Clinic criteria, $\mathrm{AD}$ according to NINDSADRDA criteria, and normal controls were classified as participants who performed within normal limits on neuropsychological testing (McKhann et al., 2011; Petersen and Negsh, 2008). All diagnoses were determined through a consensus review panel. Diagnoses of depression and diabetes were also assigned by consensus review based on self-reported medical history (including medication status), fasting blood labs (glucose and HBalc levels), and the 30-item Geriatric Depression Scale. This research was conducted under an Institutional Review Boardapproved protocol with each participant (and/or informants for cognitively impaired persons) providing written informed consent.

\section{Blood collection and biomarker analysis}

Fasting blood samples were collected on all participants according to the recently published international guidelines (O'Bryant et al., 2015). The protocol for blood collection was: (1) fasting blood collected using $21 \mathrm{~g}$ needle, (2) sample tubes collected in the following order - serum then plasma ethylenediaminetetraacetic acid (EDTA) tube, (3a) serum tubes were allowed clot for $30 \mathrm{~min}$ at room temperature in a vertical position, (3b) plasma tubes were gently inverted 5-10 times, (4) centrifuged with horizontal rotor for $10 \mathrm{~min}$ at $2,000 \times \mathrm{g}$ within $1 \mathrm{~h}$ of collection, (5) $1.0 \mathrm{~mL}$ aliquots of serum was transferred into polypropylene (cryovial) tubes, (6) sample ID was affixed to each aliquot, and (7) samples were placed into $-80^{\circ} \mathrm{C}$ freezer within $2 \mathrm{~h}$ of collection. Electronic monitoring of each aliquot (i.e. location, number, sample use) was done via Freezerworks monitoring system. Temperature monitoring of all freezers was done via the Rees Scientific system (http://www.reesscientific.com/).

Proteomic analyses were conducted via electrochemiluminescence using the Meso Scale Discovery Platform (MSD) based on our previously published protocol (O'Bryant et al., 2011). The MSD platform has been used extensively to assay biomarkers associated with a range of human diseases including $\mathrm{AD}$. In our prior work, we conducted discovery and validation studies to identify and refine a putative AD blood profile (O'Bryant et al., 2010; 2011; 2014; 2016). The AD algorithm consists of 21 proteins and has been validated across platforms, species, and tissue type. Additionally, this 21-protein $\mathrm{AD}$ algorithm retains excellent diagnostic accuracy in detecting $\mathrm{MCI}$ and $\mathrm{AD}$ among Mexican Americans (Edwards et al., 2016). The proteins included in the algorithm are as follows: fatty acid-binding protein (FABP), $\beta 2$ microglobulin, pancreatic polypeptide (PPY), macrophage inflammatory protein-1 $\alpha$, CRP, soluble vascular cell adhesion molecule-1 (sVCAM-1), thrombopoietin, $\alpha 2$ macroglobulin, eotaxin 3, tumor necrosis factor-alpha (TNF- $\alpha$ ), tenascin C (TNC), interleukin-5 (IL-5), IL-6, IL-7, IL-10, IL-18, I309, Factor VII, thymus and activation-regulated chemokine (TARC), serum amyloid A (SAA), and soluble intercellular cell adhesion molecule-1 (sICAM-1). All assays were conducted according to manufacture protocols; coefficient of variances of nearly all assays were $<10 \%$. Average values and lowest level of detection for each marker from $n=1,329$ subjects have been published elsewhere (O'Bryant et al., 2016).

\section{Statistical analyses}

The goal of the study was to examine the proteomic characteristics of Mexican Americans with comorbid depression and diabetes. Therefore, the cohort was divided into four groups: neither (neither depression nor diabetes), Depression only (depression in absence of diabetes), Diabetes only (diabetes in absence of depression), and Comorbid (both depression and diabetes). Descriptive statistics can be found in Table 1. Our proteomic profile was created using support vector machine (SVM) analyses with fivefold cross-validation with the models split by the four groups to determine the impact of depression and diabetes on the proteomic profiles as well as overall accuracy of the profile. SVM is a discriminative classifier that outputs an optimal hyperplane which categorizes new samples, given labeled training data. The advantage of fivefold cross-validation is that all the samples in the dataset are eventually used for both training and testing. The SVM model provides multiple performance measures: precision, accuracy, sensitivity, specificity, and area under the curve (AUC). The SVM model was performed using e1071 package in $\mathrm{R}$ (Version 3.3.3). The multiple performance measures are calculated as follows: precision $=\mathrm{tp} /(\mathrm{tp}+\mathrm{fp}) ;$ accuracy $=(\mathrm{tp}+\mathrm{tn}) /(\mathrm{tp}+$ $\mathrm{tn}+\mathrm{fp}+\mathrm{fn}) ;$ sensitivity $=\mathrm{tp} /(\mathrm{tp}+\mathrm{fn}) ;$ specificity $=$ $1-\mathrm{fp} /(\mathrm{fp}+\mathrm{tn}) ; \mathrm{tp}$ is true positive, fp is false positive, th is true negative, $\mathrm{fn}$ is false negative. AUC is calculated using ROCR package in $R$.

\section{Results}

This study utilized data collected from 514 Mexican Americans in the Health and Aging Brain Study 
Table 1. Demographics

\begin{tabular}{|c|c|c|c|c|}
\hline & $\begin{array}{c}\text { NEITHER (NO DEPRESSION } \\
\text { NOR DIABETES) }\end{array}$ & DEPRESSION ONLY & $\begin{array}{l}\text { DIABETES } \\
\text { ONLY }\end{array}$ & $\begin{array}{l}\text { COMORBID (DEPRESSION } \\
\text { AND DIABETES) }\end{array}$ \\
\hline & $N=184$ & $N=118$ & $N=127$ & $N=85$ \\
\hline Age & $59.77(7.65)$ & $60.55(7.57)$ & $61.68(8.35)$ & $60.02(6.90)$ \\
\hline Gender $\%$ male & $25.0 \%$ & $21.2 \%$ & $25.2 \%$ & $22.4 \%$ \\
\hline Normal control & 159 & 85 & 108 & 62 \\
\hline MCI & 25 & 33 & 19 & 23 \\
\hline Years in the U.S.A. & $38.26(19.04)$ & $34.63(18.45)$ & 37.85 (19.99) & $33.89(16.32)$ \\
\hline Primary language \% English & $24.6 \%$ & $9.84 \%$ & $19.2 \%$ & $13.7 \%$ \\
\hline \multirow[t]{2}{*}{ Education in years } & $8.93(4.46)$ & $7.09(3.88)$ & $8.05(4.59)$ & $7.01(3.70)$ \\
\hline & $0-18$ & $0-17$ & $0-20$ & $0-17$ \\
\hline
\end{tabular}

Table 2. Prediction performance for the impact of comorbid depression-diabetes on the proteomic profile of $\mathrm{MCl}$ cases

\begin{tabular}{|c|c|c|c|c|c|c|c|c|c|c|}
\hline \multirow{2}{*}{$\begin{array}{l}\text { WITHOUT } \\
\text { INTRODUCING } \\
\text { DIABETES AND } \\
\text { DEPRESSION } \\
\text { PREDICTED }\end{array}$} & \multicolumn{2}{|c|}{$\begin{array}{c}\text { WITHOUT } \\
\text { INTRODUCING } \\
\text { DIABETES AND } \\
\text { DEPRESSION }\end{array}$} & \multicolumn{2}{|c|}{ DIABETES ONLY } & \multicolumn{2}{|c|}{$\begin{array}{c}\text { DEPRESSION } \\
\text { ONLY }\end{array}$} & \multicolumn{2}{|c|}{$\begin{array}{c}\text { COMORBID } \\
\text { (DIABETES AND } \\
\text { DEPRESSION) }\end{array}$} & \multicolumn{2}{|c|}{$\begin{array}{c}\text { NEITHER } \\
\text { (NO DIABETES } \\
\text { OR DEPRESSION) }\end{array}$} \\
\hline & MCI & $\mathrm{NC}$ & MCI & $\mathrm{NC}$ & MCI & $\mathrm{NC}$ & MCI & $\mathrm{NC}$ & MCI & $\mathrm{NC}$ \\
\hline MCI & 47 & 0 & 13 & 0 & 23 & 1 & 8 & 0 & 9 & 0 \\
\hline $\mathrm{NC}$ & 39 & 329 & 7 & 87 & 4 & 71 & 10 & 51 & 12 & 119 \\
\hline Precision & \multicolumn{2}{|c|}{$100 \%$} & \multicolumn{2}{|c|}{$100 \%$} & \multicolumn{2}{|c|}{$95.83 \%$} & \multicolumn{2}{|c|}{$100 \%$} & \multicolumn{2}{|c|}{$100 \%$} \\
\hline Accuracy & \multicolumn{2}{|c|}{$90.60 \%$} & \multicolumn{2}{|c|}{$93.46 \%$} & \multicolumn{2}{|c|}{$94.95 \%$} & \multicolumn{2}{|c|}{$85.51 \%$} & \multicolumn{2}{|c|}{$91.43 \%$} \\
\hline Sensitivity & \multicolumn{2}{|c|}{0.55} & \multicolumn{2}{|c|}{0.65} & \multicolumn{2}{|c|}{0.85} & \multicolumn{2}{|c|}{0.44} & \multicolumn{2}{|c|}{0.42} \\
\hline Specificity & \multicolumn{2}{|c|}{1.0} & \multicolumn{2}{|c|}{1.0} & \multicolumn{2}{|c|}{0.99} & \multicolumn{2}{|c|}{1.0} & \multicolumn{2}{|c|}{1.0} \\
\hline AUC & \multicolumn{2}{|c|}{0.97} & \multicolumn{2}{|c|}{0.99} & \multicolumn{2}{|c|}{0.98} & \multicolumn{2}{|c|}{0.99} & \multicolumn{2}{|c|}{1.0} \\
\hline
\end{tabular}

(normal control $n=414$, MCI $n=100$ ). The average age of participants was 60 years old. Participants were primarily tested in Spanish for $406(78.8 \%)$ compared to English 109 (21.2\%) participants. The sample consisted of $393(76.3 \%)$ females and 122 (23.7) males. The marital status of the participants was $283(55.0 \%)$ married, $104(20.2 \%)$ divorced, 55 (10.7\%) widowed, $47(9.1 \%)$ separated, $23(4.5 \%)$ never married, and $3(.6 \%)$ information not available. Descriptive statistics for the four groups (Neither, Depression only, Diabetes only, and Comorbid) can be found in Table 1 .

SVM modeling was used to examine the impact of diabetes, depression, and comorbidity on our proteomic profile of MCI among Mexican Americans. Proteomic data were available from $n=414$ cases (Diabetes only $n=107$; Depression only $n=99$; Comorbid $n=69$; Neither $n=140$ ) with consensus diagnoses of MCI $(N=100)$ vs NC $(N=414)$. Table 2 provides the accuracy statistics for all models. As can be seen, our proteomic profile achieves excellent accuracy for all diagnostic groups/ comorbidities for detecting MCI among Mexican Americans with all AUCs $\geq 0.97$. Even in the context of medical comorbidities, the accuracy was at $85 \%$ at the lowest with most models $\geq 90 \%$. Specificity (SP) was 0.99-1.0 for all models; however, sensitivity $(\mathrm{SN})$ ranges from 0.42 to 0.85 . As can be seen from Table 2, the optimal balance between SN and SP was found within the Depression-only group with $\mathrm{SP}=0.99$ and $\mathrm{SN}=0.85$. With a $20 \%$ base rate of MCI among those aged 65 years and older (consistent with prior publications in community-based settings), this proteomic profile would yield a positive predictive value of 0.96 and a negative predictive value of 0.96 . There are two primary results when examining the proteomic profile variable relative importance plots (Table 3). First, the proteomic profile of MCI among Mexican Americans appears to include a heavy inflammatory component, which is consistent with our recent work specifically examining a proteomic profile of amnestic MCI (Edwards et al., 2016). Secondly, the presence of diabetes (with or without depression) introduces higher rankings of metabolic markers in the profile (e.g. PPY, FABP). However, the Depression-only proteomic profile of MCI was largely inflammatory in nature, suggesting 
Table 3. Importance scores for the impact of comorbid depression-diabetes on the proteomic profile of $\mathrm{MCl}$ cases

\begin{tabular}{|c|c|c|c|c|}
\hline $\begin{array}{l}\text { WITHOUT INTRODUCING } \\
\text { DIABETES AND DEPRESSION }\end{array}$ & DIABETES ONLY & $\begin{array}{l}\text { DEPRESSION } \\
\text { ONLY }\end{array}$ & $\begin{array}{l}\text { COMORBID (DIABETES } \\
\text { AND DEPRESSION) }\end{array}$ & $\begin{array}{l}\text { NEITHER (NO DIABETES } \\
\text { OR DEPRESSION) }\end{array}$ \\
\hline IL10 23.3 & FABP 16.2 & TNF- $\alpha 19.9$ & Eotaxin 38.0 & TNF- $\alpha 9.1$ \\
\hline TARC 20.0 & IL10 11.8 & IL10 11.9 & TARC 7.9 & B2M 9.0 \\
\hline TNF- $\alpha 19.3$ & TNF- $\alpha 8.6$ & IL7 8.2 & sVCAM1 7.0 & TPO 8.7 \\
\hline FABP 17.4 & IL6 6.3 & FVII 8.2 & TPO 5.6 & IL5 7.5 \\
\hline IL5 15.0 & TARC 6.1 & TNC 7.8 & CRP 5.2 & IL18 6.8 \\
\hline SAA 14.1 & sICAM1 5.8 & FABP 7.4 & TNF- $\alpha 4.8$ & sVCAM1 6.2 \\
\hline PPY 12.6 & PPY 4.9 & TARC 7.2 & PPY 4.5 & sICAM1 6.2 \\
\hline IL6 12.6 & TPO 4.6 & IL6 6.7 & sICAM1 4.0 & FABP 6.1 \\
\hline $\operatorname{IL} 1 \beta 12.3$ & Eotaxin3 4.6 & SAA 6.0 & IL1 $\beta 3.5$ & IL6 6.0 \\
\hline TPO 12.3 & IL1 $\beta 3.9$ & B2M 5.4 & IL5 3.5 & PPY 6.0 \\
\hline CRP 10.5 & SAA 3.7 & TPO 4.3 & SAA 3.3 & CRP 5.4 \\
\hline TNC 9.8 & B2M 3.5 & I309 3.7 & IL18 2.8 & TARC 5.3 \\
\hline B2M 7.5 & FVII 3.1 & Eotaxin3 3.6 & TNC 2.5 & Eotaxin3 4.4 \\
\hline Eotaxin3 7.4 & IL18 2.7 & sVCAM1 2.5 & IL6 2.4 & IL1 $\beta 3.6$ \\
\hline sVCAM1 6.3 & I309 2.6 & IL1 $\beta 2.4$ & IL7 2.4 & IL10 3.3 \\
\hline IL7 6.0 & CRP 2.5 & IL18 2.2 & B2M 1.7 & IL7 2.6 \\
\hline sICAM1 5.9 & IL5 1.9 & IL5 2.0 & FVII 1.7 & SAA 2.4 \\
\hline FVII 5.5 & TNC 1.4 & sICAM1 0.9 & IL10 1.3 & TNC 2.3 \\
\hline I309 4.8 & sVCAM1 1.1 & CRP 0.8 & I309 0.7 & A2M 1.9 \\
\hline IL18 2.0 & IL7 0.6 & A2M 0.5 & FABP 0.1 & I309 1.8 \\
\hline A2M 0.6 & A2M 0.1 & PPY 0.4 & A2M 0.1 & FVII 0.7 \\
\hline
\end{tabular}

that medical comorbidities may not have tremendous impact on the overall accuracy of our proteomic profile, but the pathological mechanisms may vary and be additive in nature.

\section{Discussion}

Prior research has shown that both depression and diabetes impact cognitive function. The purpose of the current study was to investigate the impact of depression and diabetes on the proteomic profiles of individuals with comorbid depression and diabetes, Depression only, Diabetes only, or Neither condition. This study found that Mexican Americans with MCI who had different comorbidities exhibited distinct biomarker profiles. The biomarker profile for MCI in the absence of depression or diabetes was predominately inflammatory; the biomarker profile for the presence of diabetes alone was primarily metabolic; the profile for depression alone was largely inflammatory; and the biomarker profile for the presence of both comorbidities included cardiovascular risk markers (ICAM, CRP).

Analysis of our previously established blood profile for detecting $\mathrm{AD}$ revealed that neither comorbidity had a substantial impact on the overall accuracy of the algorithm itself. That is, the presence of diabetes, depression or both only minimally impacted the overall accuracy of the algorithm.
However, while specificity remained excellent across groups, there was an impact on sensitivity. Additionally, the relative importance of the proteins within the algorithm changed by group (None, Depression only, Diabetes only, Comorbid). As has been noted in the literature, MCI is a heterogeneous categorization with multiple causes. Our results suggest that MCI due to metabolic dysfunction may be a novel subgroup biologically, whereas depression due to inflammatory dysfunction may be a biologically distinct group. In our prior work which was based on a cohort that used depression as an exclusion criteria, we demonstrated that the proteomic profile of $\mathrm{AD}$ was more metabolic in nature among Mexican Americans as compared to an inflammatory/vascular-driven profile among non-Hispanic whites (O'Bryant et al., 2014). The current results suggest the need for a biological stratification of MCI cases for a more comprehensive understanding of underlying causes of cognitive dysfunction. The biological stratification may give providers the tools to know which patients may benefit from treatment with an antidepressant to impact cognitive function.

There are several weaknesses worth noting in this study. First, this is an epidemiological study and not a clinic-based study and, therefore, the number of individuals in cognitive dysfunction groups was relatively small and the sample was predominantly female. While the cognitively impaired sample is a 
reflection of the base rate in the community, a larger more gender equivalent sample would strengthen the study. We are currently conducting a more comprehensive study to include $n=1,000$ community-dwelling Mexican Americans and $n=1,000$ non-Hispanic whites, and the current work will be expanded within that cohort. Additionally, the current analyses are cross-sectional in nature. However, the ongoing work of the team will capture longitudinal proteomic data for additional analyses to assess change over time. Despite these limitations, this is the first-ever comprehensive examination of diabetes-depression comorbidity proteomic profiles among community-dwelling Mexican Americans. Medical comorbidities impact the proteomic profiles indicative of MCI, which is suggestive of multiple biological dysfunction and can set the stage for additional investigations into the viability of a precision medicine approach to treating and preventing MCI among this underserved population. Additionally, the current findings highlight the need to fully examine medical comorbidities individually and in combination in order to better understand the factors contributing to $\mathrm{MCI}$ among Mexican Americans.

\section{Conflicts of interest}

Dr. O'Bryant has pending patents related to his Alzheimer's disease blood test. UNTHSC has licensed these patents to CX Precision Medicine (CX PM), Inc. Dr. O'Bryant has a financial interest in this company and is the Chief Scientific Advisor. Dr. Johnson has a financial interest in CX PM. CX PM had no role in the design or results of this study.

\section{Source of funding}

Research reported in this publication was supported by the National Institute on Aging under Award Numbers R01AG054073, R01AG051848, R01AG058537, R01AG058252, and R56AG 054073. The National Institutes of Health had no role in the design and conduct of the study: collection, management, analysis, and interpretation of the data; and preparation, review, or approval of the manuscript; and decision to submit the manuscript for publication. The content is solely the responsibility of the authors and does not necessarily represent the official views of the National Institutes of Health. Additional support was provided by the Alzheimer's Association NIRG, AARG-16-442652, and the JES Edwards foundation. The study team would like to thank the HABLE study participants and Fort Worth community for supporting this study.

\section{Description of authors' roles}

Conceived and designed the experiments: LAJ, FZ, SEL, JRH, and SO. Performed the experiments: LAJ, FZ, SEL, JRH, and SO. Analyzed the data: LAJ, SEL, and FZ. Contributed reagents/materials/analysis tools: LAJ, SEL, FZ, JRH, and SO. Wrote the manuscript: LAJ, SEL, FZ, JRH, and SO. Other: ICMJE criteria for authorship read and met: LAJ, SEL, FZ, $\mathrm{JRH}$, and SO. Agree with manuscript results and conclusions: LAJ, SEL, FZ, JRH, and SO.

\section{References}

Alzheimer's Association. (2017). Alzheimer's disease facts and figures. Alzheimer's Disease Facts and Figures, 13, 325-373. doi: 10.1016/j.jalz.2017.02.001.

Arvanitakis, Z., Wilson, R.S, Bienias, J.L., Evans, D.A. and Bennett, D.A. (2004). Diabetes mellitus and risk of Alzheimer disease and decline in cognitive function. Archives of Neurology, 61, 661-666. doi: 10.1001/archneur .61.5.661.

Barnes, D.E., Alexopoulos, G.S., Lopez, O.L., Williamson, J.D. and Yaffe, K. (2006). Depressive symptoms, vascular disease, and mild cognitive impairment: Findings from the Cardiovascular Health Study. Archives of General Psychiatry, 63, 273-279. doi: 10 .1001/archpsyc.63.3.273.

de la Monte, S.M. (2014). Type 3 diabetes is sporadic Alzheimer's disease: Mini-review. European Neuropsychopharmacology, 24, 1954-1960. doi: 10.1016/j .euroneuro.2014.06.008

Downer, B., Chen, N.W., Wong, R. and Markides, K.S. (2016). Self-reported health and functional characteristics of Mexican and Mexican American adults aged 80 and over. fournal of Aging and Health, 28, 1239-1255. doi: 10.1177/ 0898264316656508.

Edwards, M., Hall, J., Williams, B., Johnson, L. and O'Bryant, S. (2016). Molecular markers of amnestic mild cognitive impairment among Mexican Americans. Fournal of Alzheimer's Disease, 49, 221-228. doi: 10.3233/jad-150553.

Elias, M.F., Elias, P.K., Sullivan, L.M., Wolf, P.A. and D'Agostino, R.B. (2005). Obesity, diabetes and cognitive deficit: The Framingham Heart Study. Neurobiology of Aging, 26, S11-S16. doi: 10.1016/j.neurobiolaging.2005.08.019.

Gerst, K., AL-Ghatrif, M., Beard, H.A., SamperTernent, R. and Markides, K.S. (2010). High depressive symptomatology among older community-dwelling Mexican Americans: The impact of immigration. Aging and Mental Health, 14, 347-354. doi: 10.1080/ 13607860903292578.

González, H.M., Wassim, T., Whitfield, K.E. and Vega, W.A. (2010). The epidemiology of major depression and ethnicity in the United States. Fournal of Psychiatric Research, 44, 1043-1051. doi: 10.1016/j.jpsychires.2010. 03.017.

Haan, M.N., Mungas, D.M., Gonzalez, H.M., Ortiz, T.A., Acharya, A. and Jagust, W.J. (2003). Prevalence of dementia in older latinos: The influence of type 2 diabetes mellitus, stroke and genetic factors. Fournal of the American 
Geriatrics Society, 51, 169-177. doi: 10.1046/j.1532-5415 .2003.51054.x.

Hinton, L. et al. (2012). Falling through the cracks: Gaps in depression treatment among older Mexican-origin and white men. International fournal of Geriatric Psychiatry, 27, 1283-1290. doi: 10.1002/gps.3779.

Jacobsen, L.A., Kent, M., Lee, M. and Mather, M. (2011). America's aging population. Population Bulletin, 66, 1-20. https://www.prb.org/americas-aging-population/.

Johnson, L.A. et al. (2015). Comorbid depression and diabetes as a risk for Mild Cognitive Impairment and Alzheimer's Disease in elderly Mexican Americans. Fournal of Alzheimer's Disease, 129-136. doi: 10.3233/ jad-142907.

Kandimalla, R., Thirumala, V. and Reddy, P.H. (2017) Is Alzheimer's disease a Type 3 Diabetes? A critical appraisal. Biochimica et Biophysica Acta, 1863, 1078-1089. doi: 10.1016/j.bbadis.2016.08.018.

Luchsinger, J.A., Reitz, C., Patel, B., Tang, M., Manly, J.J. and Mayeux, R. (2007). Relation of diabetes to mild cognitive impairment. Archives of Neurology, 64, 570-575. doi: 10.1001/archneur.64.4.570.

McKhann, G.M. et al. (2011). The diagnosis of dementia due to Alzheimer's disease: Recommendations from the National Institute on Aging-Alzheimer's Association workgroups on diagnostic guidelines for Alzheimer's disease. Alzheimers E Dementia, 7, 263-269. doi: 10.1016/j .jalz.2011.03.005.

Modrego, P.J. and Ferrandez, J. (2004). Depression in patients with mild cognitive impairment increases the risk of developing dementia of Alzheimer type: A prospective cohort study. Archives of Neurology, 61, 81290-1293. doi: 10.1001/archneur.61.8.1290.

Novak, K. and Riggs, J. (2004). Hispanics/Latinos and Alzheimer's disease. Alzheimer's Association, 1-8. https:// www.alz.org/media/Documents/alzheimers-hispanicslatinos-r.pdf.

O'Bryant, S.E. et al. (2010). A serum protein-based algorithm for the detection of Alzheimer disease. Archives of Neurology, 67, 1077-1081. doi: 10.1001/archneurol. 2010.215.

O'Bryant, S.E. et al. (2011). A blood-based screening tool for Alzheimer's disease that spans serum and plasma: Findings from TARC and ADNI. PLoS One, 6, e28092. doi: 10.1371/journal.pone.0028092.

O'Bryant, S.E. et al. (2013a). Characterization of Mexican Americans with mild cognitive impairment and Alzheimer's disease. Fournal of Alzheimer's Disease, 33, 373-379. doi: 10 $.3233 /$ jad-2012-121420.

O'Bryant, S.E. et al. (2013b). Risk factors for mild cognitive impairment among Mexican Americans. Alzheimer's $\mathcal{E}$ Dementia, 9, 622-631, e621. doi: 10.1016/j.jalz.2012. 12.007.

O'Bryant, S.E. et al. (2013c). Biomarkers of Alzheimer's disease among Mexican Americans. Fournal of Alzheimer's Disease: $\mathcal{F A D}, 2013$, 34, 841-849. doi: 10.3233/jad-122074.

O'Bryant, S.E. et al. (2013d) The link between C-reactive protein and Alzheimer's disease among Mexican
Americans. Fournal of Alzheimer's Disease, 34, 701-706. doi: $10.3233 /$ jad-122071.

O'Bryant, S.E. et al. (2014). Validation of a serum screen for Alzheimer's disease across assay platforms, species, and tissues. Fournal of Alzheimer's Disease, 42, 1325-1335. doi: $10.3233 /$ jad-141041.

O'Bryant, S.E. et al. (2015). Guidelines for the standardization of preanalytic variables for blood-based biomarker studies in Alzheimer's disease research. Alzheimer's \& Dementia, 11, 549-560. doi: 10.1016/j.jalz. 2014.08.099.

O'Bryant, S.E. et al. (2016). A blood screening test for Alzheimer's disease. Alzheimer's E Dementia (Amsterdam, Netherlands), 3, 83-90. doi: 10.1016/j.dadm.2016.06.004.

O'Bryant, S.E., Humphreys, J.D., Sutker, P.B. and Schiffer, R.B. (2007). Presentation of Mexican American patients to a memory disorder clinic. Fournal of Psychopathology and Behavioral Assessment, 29, 137-140. doi: 10.1007/s10862-006-9042-9.

Ott, A., Stolk, R.P., Hofman, A., van Harskamp, F., Grobbee, D.E. and Breteler, M.M. (1996). Association of diabetes mellitus and dementia: The Rotterdam study. Diabetolgia, 39, 1392-1397. doi: 10.1007/s001250050588.

Ott, A., Stolk, R.P., van Harskamp, F., Pols, H.A.O., Hofman, A. and Breteler, M.M. (1999). Diabetes mellitus and the risk of dementia: The Rotterdam study. Neurology, 53, 1937-1942. doi: 10.1212/wnl.53.9.1937.

Palmer, B.W., Boone, K.B., Lesser, I.M., Wohl, M.A., Berman, N. and Miller, B.L. (1996). Neuropsychological deficits among older depressed patients with predominantly psychological or vegetative symptoms. fournal of Affective Disorders, 41, 17-24. doi: 10.1016/ 0165-0327(96)00059-6.

Peila, R., Rodriguez, B. and Launer, L.J. (2002). Type 2 diabetes, APOE gene, and the risk for dementia and related pathologies: The Honolulu-Asia Aging study. Diabetes, 51, 1256-1262. doi: 10.2337/diabetes.51.4.1256.

Petersen, R.C. and Negsh, S. (2008). Mild cognitive impairment: An overview. CNS Spectrums, 13, 45-53. doi: $10.1017 /$ s 1092852900016151 .

Smith, K.J., Au, B., Ollis, L. and Schmitz, N. (2018). The association between C-reactive protein, Interleukin- 6 and depression among older adults in the community: A systematic review and meta-analysis. Experimental Gerontology, 102, 109-132. doi: 10.1016/j.exger.2017.12 .005 .

Sundquist, J. and Winkleby, M.A. (1999). Cardiovascular risk factors in Mexican American adults: A transcultural analysis of NHANES III, 1988-1994. American Fournal of Public Health, 89, 723-730. doi: 10.2105/ ajph.89.5.723.

Tiemeier, H., Hofman, A., Van Tuijl, H., Kiliaan, A., Meijer, J. and Breteler, M. (2013). Inflammatory proteins and depression in the elderly. Epidemiology, 14, 103-107. doi: 10.1097/00001648-200301000-00025.

Verdile, G., Fuller, S.J. and Martins, R.N. (2015). The role of type 2 diabetes in neurodegeneration. Neurobiology of Disease, 84, 22-38. doi: 10.1016/j.nbd.2015.04.008. 